\title{
Knowledge and attitudes of physiotherapy students towards obesity
}

\begin{abstract}
Obesity has been recognised as a risk factor for non-communicable diseases, with more than one billion adults worldwide who are overweight, of which approximately 300 million are obese. Obesity puts an individual in danger of a shorter life expectancy and at risk for developing chronic diseases of lifestyle, which include diabetes, cardiovascular disease and musculoskeletal disorders such as arthritis and back pain. Widespread negative attitudes towards obese people have been observed which are equally prevalent among health care professionals. This study was based on the need to determine the knowledge and attitudes towards obese people among physiotherapy students, as they are well suited to address the intricacies of obesity and its related conditions. One hundred and seventy five students from a university in the Western Cape, South Africa, completed a structured, self-administered questionnaire that was adopted from the Obesity Risk Knowledge and Fat Phobia Scale. The study sample demonstrated average levels of knowledge regarding obesity with scores ranging from 3 to 9 on a scale of 10 with a mean score of 6.05. An overwhelming majority of the participants $(>80 \%)$ viewed obesity as largely a behavioural problem while nearly all the participants (97.6\%) characterised obese people as lazy, unattractive, insecure and with lower self-esteem. This study has reinforced the need for a more focussed approach to the education of physiotherapy students around obesity and obesity-related conditions, as well as the management thereof.
\end{abstract}

KEYWORDS: OBESITY, ATTITUDES, KNOWLEDGE, PHYSIOTHERAPY, STUDENTS.

\section{INTRODUCTION}

The high prevalence of obesity and its strong link with major predictors of a number of life-threatening and debilitating conditions has made it a subject of considerable and primary concern on the agenda of the public health (U S Department of Health and Human Services, 2001). The World Health Organisation recognises obesity as a risk factor for non-communicable diseases in which more than one billion adults worldwide are overweight, of which approximately 300 million are obese (World Health Organisation, 2006). Its prevalence in Western nations such as the United Sates is as high as $27 \%$ in men and $32 \%$ in women above the age of 20 years (World Health Organisation, 2006). However, the burden of being overweight and obese is not only to be found in the Western world, as countries undergoing economic transition such as South Africa, Brazil, and China have also been implicated (Pouane et al., 2002). Pouane et al. (2002) estimated that the prevalence of overweight and obese people in South Africa is high with more than $29 \%$ of men and $56 \%$ of women being classified as overweight or obese. The impact of obesity on the health of individuals is critical, as it puts the individual in danger of a shorter life expectancy and at risk for developing chronic diseases of lifestyle such as diabetes, cardiovascular diseases and musculoskeletal disorders, including arthritis and back pain (Canadian Physiotherapy Association, 2008).

Various researchers show that the rapid increase in the prevalence of obesity is not only due to behavioural and lifestyle problems as previously believed but also in part to biological, genetic and hormonal influence (Snyder et al., 2004). This raises a concern when public health and clinical interventions focus exclusively on lifestyle without acknowledging other causes of obesity (Thomas, Hyde, \& Komesaroff, 2007). It is now increasingly evident that individuals may be overwhelmed by the complex factors involved in the causes of obesity (Rosmond, 2004). In addition, obesity is erroneously believed to be under the personal control of the indi- vidual, the consequence of which often lead to blame and judgement among healthcare providers. Some studies have attributed this perception to a deficiency in training programmes and limited opportunities for further studies in obesity-related knowledge (Block, Desalvo \& Fisher, 2003).

Some studies have reported this negative attitude and other forms of stereotyping among groups of healthcare professionals, including nurses (Maroney \& Golub, 1992), and physicians (Loomis, Connolly, Clinch, \& Djuric, 2001). Students in the areas of dietetics (Berryman, Dubale, Manchester \& Mittelstaedt, 2006) and exercise science (Chambliss, Finley, \& Blair, 2004) have also been reported to have negative attitudes towards obese people.

\section{Correspondence to:}

JS Phillips (PhD) Physiotherapy

Associate Professor

Department of Physiotherapy

University of the Western Cape

Email:jphillips@uwc.ac.za 
There is a concern that this negative attitude towards people who are obese among the healthcare professionals will not only compromise their clinical judgements but also dissuade obese clients from seeking medical help (Puhl \& Brownell, 2001). Furthermore, the consequence of such negative perception will also result in the under-utilization of healthcare services and increased obesity mortality (Thomas et al., 2007).

Obese and overweight individuals experience a number of problems which may contribute to a lack of function, such as muscle weakness, joint pain, difficulty with walking and climbing stairs (Peltonen, Lindroos \& Torgerson, 2003). As primary health care professionals, physiotherapists are ideally suited to individualize exercise regimens within the functional limitations of obese patients (Canadian Physiotherapy Association, 2008). Furthermore physiotherapists have received training in therapeutic exercises, biomechanics, exercise prescription and physiological and anatomical mechanisms of health and disease. Various physiotherapy associations are in agreement that due to these varied skill sets physiotherapist are well suited to address the intricacies of obesity and its related conditions (Australian Physiotherapy Association, 2009; Canadian Physiotherapy Association, 2008). For physiotherapists to do this effectively, they must be able to recognize the complex nature of obesity aetiology and be aware of their attitudes towards obese individuals. Therefore, the motivation for this study was based on the need to determine the knowledge and attitudes towards obese people among physiotherapy students.

\section{METHODS}

The study employed a cross-sectional, quantitative design. The population included 220 full time undergraduate and postgraduate physiotherapy students registered for the 2008 academic year at a university in the Western Cape, South Africa. Of these students, 79.5\% were undergraduates and $20.5 \%$ were postgraduates. All students were invited to participate in the study.

Permission and ethical clearance were granted by the relevant authorities.
The questionnaires were administered to participants in a class setting. At the beginning of each session the purpose of the study was clearly explained by the researcher to the participants. Signed, informed consent was obtained from all the participating students and an information sheet provided, explaining their right of voluntary participation, confidentiality and withdrawal as entrenched in a standard research protocol.

Data were collected by means of a structured, self administered questionnaire consisting of two scales for measuring knowledge and attitudes towards obese people. The first scale, Obesity Risk Knowledge (ORK-10), developed and validated by Swift, Glazebrook, and Macdonald (2006) was used to measure the knowledge of the health risks associated with obesity. It is designed to be a 'norm referenced' as opposed to 'criteria referenced', which means that there is no cut-off point to say that knowledge is either high or low. Scores on the ORK-10 scale ranges between 0 and 10 with higher scores indicating higher levels of knowledge. The second scale, the Fat Phobia Scale (FPS) was developed and validated by Bacon, Scheltema, and Robinson (2001), and was used to determine students' attitudes towards obese people. The FPS consists of 14 items using a 5-point Likert scale ranging from 1 to 5 . Based on the score design, a score of 2.5 indicates neutral attitudes towards obese individuals. A score of more or less than 2.5 indicates a more negative or positive attitude respectively.

Data obtained from the questionnaire were analyzed using the Statistical Package for the Social Sciences (SPSS) version 16.0. Descriptive statistics were employed to summarize the data using frequency tables and were expressed as percentages, means, and standard deviations. The differences between socio-demographic variables and specific variables (knowledge and attitudes) were tested using the Chi-square. The correlation co-efficient was used to test the strength of the relationship between knowledge and attitudes and the alpha level was set at $\mathrm{p}<0.05$.

\section{RESULTS}

One hundred and seventy five (175) participants completed and returned the questionnaires. The authors decided not to include questionnaires in the analysis if more than $50 \%$ of the items were incomplete. Five questionnaires were thus considered invalid. The overall response rate was thus $77.3 \%$. The final sample consisted of $73.5 \%(n=125)$ females and $26.5 \%(n=45)$ males. The participants' age ranged from 17-49 years with a mean age of 21.5 years $(\mathrm{SD}=4.9)$. More than half of the participants $(56 \%)$ had a normal weight using a self-reported body weight and height for body mass index (BMI). Overall, the majority of the study participants $(85 \%)$ reported that they did not receive education regarding obesity and about $23 \%$ were confident in treating and counselling obese clients.

\section{KNOWLEDGE OF OBESITY}

The scores of the study sample based on the ORK-10 ranged from 3 to 9 (mean score $=6.08, \mathrm{SD}=1.35)$. More than half of the participants $(51.1 \%)$ achieved scores of 5 and 6 on a scale of 10 . Table 1 presents the responses to the general knowledge questions (ORK-10) concerning the health risks that are associated with obesity. Participants could select 'True' or 'False' for the different statements. The majority of the participants $(96.5 \%)$ knew that obesity is a risk factor for hypertension. Furthermore, $89 \%$ of the participants agreed that there is a health benefit for obese individuals to lose weight and $67.1 \%$ of the participants underestimated the risk of breast cancer after menopause in obese individuals.

The association between knowledge items and gender $\left(\chi^{2}=0.78, \mathrm{p}>0.05\right)$ and year of study $\left(\chi^{2}=0.78, p>0.05\right)$ was very low and not statistically significant. Very few individual knowledge questions had significant associations with the year of study as illustrated in Table 2 . There was a significant association between the year of study and the notion that obesity increases the risk of getting bowel cancer with postgraduate participants less likely to recognize this $\left(\chi^{2}=17.18, \mathrm{P}<0.05\right)$. Furthermore, participants in their final year were more 
Table 1: Knowledge of health risks associated with obesity of study sample $(n=170)$.

\begin{tabular}{|l|l|l|}
\hline Questions & Correct (\%) & Incorrect (\%) \\
\hline Obesity does not increase the risk of developing high blood pressure (False) & 96.5 & 3.5 \\
\hline Obese people can expect to live as long as non-obese People (False) & 90.0 & 10.0 \\
\hline $\begin{array}{l}\text { There is no health benefit if an obese person who gets diabetes, } \\
\text { loses weight (False) }\end{array}$ & 89.4 & 10.6 \\
\hline Obesity increases the risk of getting food allergy (False) & 77.2 & 22.8 \\
\hline Obesity increases the risk of getting bowel cancer (True) & 65.9 & 34.1 \\
\hline $\begin{array}{l}\text { A person with a 'beer belly' shaped stomach has an increased risk } \\
\text { of getting diabetes (True) }\end{array}$ & 65.7 & 34.3 \\
\hline $\begin{array}{l}\text { It is better for a person's health to have fat around the hips and thighs } \\
\text { than around the stomach \& waist (True) }\end{array}$ & 54.7 & 45.3 \\
\hline $\begin{array}{l}\text { Obesity increases the risk of getting breast cancer after the } \\
\text { menopause (True) }\end{array}$ & 32.9 & 67.1 \\
\hline $\begin{array}{l}\text { An obese person who gets diabetes needs to lose at least 40\% } \\
\text { of their body weight for clear health benefits (False) }\end{array}$ & 24.3 \\
\hline $\begin{array}{l}\text { Obesity is more of a risk to health for people from South-Asia (e.g. India) } \\
\text { than it is for White Europeans (True) }\end{array}$ & 14.1 & 85.9 \\
\hline
\end{tabular}

All responses expressed as percentages.

Table 2: Associations between the year of study and knowledge of health risks of obesity $(n=170)$.

\begin{tabular}{|c|c|c|c|c|c|}
\hline & Yr. 1 & Yr. 2 & Yr.3 & Yr.4 & PG (\%) \\
\hline \multicolumn{6}{|c|}{ A person with a 'beer-belly' shaped stomach has an increased risk of getting diabetes. } \\
\hline True (correct response) & 70.7 & 67.7 & 65.9 & 66.7 & 47.1 \\
\hline False & 29.3 & 32.3 & 34.1 & 33.3 & 52.9 \\
\hline \multicolumn{6}{|c|}{ Obesity increases the risk of getting bowel cancer.** } \\
\hline True (correct response) & 75.6 & 74.2 & 71.8 & 61.5 & 23.5 \\
\hline False & 24.4 & 25.8 & 28.2 & 38.5 & 76.5 \\
\hline \multicolumn{6}{|c|}{$\begin{array}{l}\text { An obese person who gets diabetes needs to lose at least } 40 \% \text { of their body weight } \\
\text { for clear health benefits. }\end{array}$} \\
\hline True & 85.4 & 63.3 & 82.9 & 65 & 82.4 \\
\hline False (correct response) & 14.6 & 36.7 & 17.1 & 35 & 17.6 \\
\hline \multicolumn{6}{|c|}{ Obese people can expect to live as long as non-obese person. ${ }^{\star \star}$} \\
\hline True & 4.9 & 16.1 & 4.9 & 5 & 35.3 \\
\hline False (correct response) & 95.1 & 83.9 & 95.1 & 95 & 64.7 \\
\hline \multicolumn{6}{|c|}{ Obesity increases the risk of getting breast cancer after menopause. } \\
\hline True (correct response) & 29.3 & 31 & 35.9 & 36.8 & 29.4 \\
\hline False & 70.7 & 69 & 64.1 & 63.2 & 70.6 \\
\hline \multicolumn{6}{|c|}{$\begin{array}{l}\text { Obesity is more of a risk to health for people from South-Asia (e.g. India and Pakistan) } \\
\text { than it is for White European.** }\end{array}$} \\
\hline True (correct response) & 4.9 & 12.9 & 14.6 & 12.5 & 41.2 \\
\hline False & 95.1 & 87.1 & 82.9 & 87.5 & 58.8 \\
\hline \multicolumn{6}{|c|}{ There is no major health benefit if an obese person who gets diabetes loses weight. } \\
\hline True & 9.8 & 19.4 & 7.3 & 5 & 17.6 \\
\hline False (correct response) & 90.2 & 80.6 & 92.7 & 95 & 82.4 \\
\hline \multicolumn{6}{|c|}{ Obesity does not increase the risk of developing high blood pressure ${ }^{\star *}$} \\
\hline True & 0 & 0 & 0 & 5 & 23.5 \\
\hline False (correct response) & 100 & 100 & 100 & 95 & 76.5 \\
\hline \multicolumn{6}{|c|}{ It is better for a person's health to have fat around the hips and thighs than around the stomach and waist.* } \\
\hline True (correct response) & 43.9 & 51.6 & 41.5 & 75 & 70.6 \\
\hline False & 56.1 & 48.4 & 58.5 & 25 & 29.4 \\
\hline \multicolumn{6}{|c|}{ Obesity increases the risk of getting a food allergy. } \\
\hline True & 31.7 & 16.7 & 9.8 & 28.2 & 18.8 \\
\hline False (correct response) & 68.3 & 83.3 & 85.4 & 71.8 & 81.2 \\
\hline
\end{tabular}

* Significant differences between groups at $\mathrm{P}<0.05$. 
likely to state correctly that it is better to for a person's health to have fat around the hip and thighs than around the stomach and waist $\left(\chi^{2}=13.33, \mathrm{P}<0.05\right)$.

\section{ATTITUDES TOWARDS OBESITY}

A substantial number of respondents (97.6\%) had a score that was greater than 2.5, and approximately $2.4 \%$ achieved a score of less than 2.5 on the Fat Phobia Scale. Table 3 summarizes the mean scores of the study sample based on the Fat Phobia Scale. Assessing individual attitude items on the Fat Phobia scale found that the majority of participants (> 60\%) thought of obese people as being slow and insecure, having low self-esteem and poor self-control, were lazy, and unattractive.

A significant difference was found between attitude items and gender, with females more likely to characterize obese individuals as having poor selfcontrol $\left(\chi^{2}=11.69, \mathrm{p}<0.05\right)$ and low self-esteem $\left(\chi^{2}=17.86, \mathrm{p}<0.05\right)$. Furthermore, the association between the participants own body mass index (BMI) and attitudes showed that significantly more underweight participants (77\%) and normal weight participants (75.8\%) were more likely to view obese people as having no endurance $\left(\chi^{2}=14.79, \mathrm{p}<0.05\right)$ and self-indulgent $\left(\chi^{2}=12.41, \mathrm{p}<0.05\right)$.

\section{DISCUSSION}

There is a growing concern that despite healthcare professionals recognizing obesity as a life-threatening medical condition, they are often confused about the effectiveness of their interventions and advice. Several studies have concluded that lack of knowledge of the causes of obesity among healthcare professionals is known to mitigate the capacity to effectively intervene or advise (Block et al., 2003). The present study highlights that physiotherapy students demonstrated average levels of knowledge regarding obesity with scores ranging from 3 to 9 on a scale of 10 with a mean score of 6.05. This classification was based on the Obesity Risk Knowledge (ORK-10) with higher scores indicating professionals to be in better positions to direct the future of obesity prevention and intervention

Table 3: Mean scores (SD) of the study samples on the Fat Phobia Scale $(n=170)$.

\begin{tabular}{|l|l|l|}
\hline Characteristics & Mean & SD \\
\hline Gender & & \\
Male & 3.84 & 0.70 \\
Female & 3.99 & 0.55 \\
\hline Year of study & & \\
Year 1 & 4.124 & 0.51 \\
Year 2 & 4.08 & 0.688 \\
Year 3 & 3.92 & 0.09 \\
Year 4 & 3.80 & 0.49 \\
Post graduate & 3.69 & 0.59 \\
\hline Body Mass Index & & \\
Underweight & 4.14 & 0.73 \\
Normal & 3.95 & 0.55 \\
Overweight & 3.91 & 0.49 \\
Obese & 3.69 & 0.59 \\
\hline Overall sample score $\mathbf{( n = 1 7 0 )}$ & 3.95 & 0.60 \\
\hline
\end{tabular}

(Swift et al., 2006). The success of their role in obesity prevention and intervention depends on their ability to recognize the consequence of obesity and to show a willingness to engage in obesity interventions (Kristeller \& Hoerr, 1997).

The levels of knowledge demonstrated by the study sample are consistent with the study by Swift, Sheard and Rutherford (2007) conducted in the United Kingdom, which found an average level of knowledge among medical students $($ mean $=6.5)$, postgraduate nursing students $($ mean $=6.0$ ) and undergraduate nursing students (mean $=5.7)$. The results of the study by Swift et al. (2007) and that of the present study indicate that students may not be well equipped to offer advice to obese patients as they do not have adequate knowledge regarding obesity. These findings confirm that a gap in students' knowledge exists. A more focused approach regarding the role of physiotherapists in the prevention and management of obesity and its associated conditions from undergraduate to postgraduate levels thus seems warranted to improve students' knowledge.

Studies have consistently shown that negative attitudes towards obese people among healthcare professionals increase the tendency for obesity-related behaviours and will likely result in differen- tial treatment that will affect equal access to healthcare facilities (Puhl \& Brownell, 2003). The current study highlighted the fact that a substantial number of participants (97.6\%) possess negative attitudes towards obese people. This is consistent with several studies among healthcare professionals who describe obese people as lazy, lacking self-control, over-indulgent, and less attractive (Loomis et al., 2001). This result is of great concern in that the vast majority of physiotherapy students failed to recognise that obesity is a product of many factors rather than predominantly a behavioural problem, thus putting the blame mainly on obese patients. This again highlights the fact that education related to obesity for physiotherapy students should be more focussed on their role in the management of obesity and its associated conditions. This is important as there is evidence of the effectiveness of physiotherapy management in musculoskeletal conditions (Altman, Hochberg, Moskowitz \& Schnitzer, 2000), type 2 diabetes (Aas et al., 2005) and respiratory problems (Satta, 2000) in the obese individual.

The current study explores the influence of the knowledge of physiotherapy students around issues regarding obesity and how it affects their attitudes towards 
people who are obese. The study demonstrated that the relationship between knowledge of the causes of obesity and attitudes towards obese people was not significant. This finding concurs with the study of Fogelman et al. (2002), which found that physicians were aware of the risk factors for obesity, but that such knowledge was not enough to improve their attitudes. This could be interpreted that students had limited knowledge about the causes of obesity. Therefore, they were less likely to express positive attitudes towards obese individuals if they view the cause of obesity to be largely within the control of the obese person.

\section{CONCLUSION}

This study has reinforced the need for a more focused approach to the education of physiotherapy students related to obesity and obesity related conditions, and the management thereof. Education should also emphasise the vital role of physiotherapy in the management and treatment of obese individuals.

\section{REFERENCES}

Aas AM, Bergstad I, Thorsby PM, Johannesen O, Solberg M \& Birkeland KI 2005 An intensified lifestyle intervention programme may be superior to insulin treatment in poorly controlled Type 2 diabetic patients on oral hypoglycaemic agents: Results of a feasibility study. Diabetic Medicine, 22: 316-322.

Altman RD, Hochberg MC, Moskowitz RW \& Schnitzer TJ 2000 Recommendations for the medical management of osteoarthritis of the hip and knee: 2000 update: American College of Rheumatology Subcommittee on Osteoarthritis Guidelines. Arthritis \& Rheumatism, 43, 1905-1915.

Australian Physiotherapy Association 2009. Australian Physiotherapy Association: Inquiry into Obesity in Australia.

Bacon JG, Scheltema KE, \& Robinson BE 2001. Fat phobia scale revisited: The short form. International Journal of Obesity, 25, 252-257.

Berryman DE, Dubale GM, Manchester DS, \& Mittelstaedt R 2006 Dietetics students possess negative attitudes toward obesity similar to nondietetics students. Journal of the American Dietetic Association, 106, 16781682 .
Block JP, Desalvo KB, \& Fisher WP 2003. Are physicians equipped to address the obesity epidemic? Knowledge and attitudes of internal medicine residents. Preventive Medicine, 36(6), 669-675.

Canadian Physiotherapy Association. 2008. Position statement: Physiotherapists and management of obesity. Retrieved March 15, 2008, from www.physiotherapy.ca

Chambliss HO, Finley CE, \& Blair SN 2004 Attitudes toward obese individuals among exercise science students. Medicine and Science in Sports and Exercise, 36(3), 468-474.

Fogelman Y, Vinker S, Lachter J, Biderman A, Itzhak B, \& Kitai E 2002 Managing obesity: A survey of attitudes and practices among Israeli primary care physicians. International Journal of Obesity Related Metabolic Disorder, 26, 1393-1397.

Kristeller JL, \& Hoerr RA 1997 Physician attitudes towards managing obesity: Differences among six specialty groups. Preventive Medicine, 26(4), 542-549.

Loomis GA, Connolly KP, Clinch CR, \& Djuric DA 2001 Attitudes and practices of military physicians regarding obesity. Military Medicine, 166, 121-125.

Maroney D, \& Golub S 1992. Nurses' attitudes toward obese persons and certain ethnic groups. Perceptual Motor Skills, 75, 387-391.

Peltonen M, Lindroos AK \& Torgerson JS 2003 Musculoskeletal pain in the obese: a comparison with a general population and long-term changes after conventional and surgical obesity treatment. Pain, 104, 549-557.

Pouane T, Steyn K, Bradshaw D, Laubscher R, Fourie J, Lambert V, Mbananga N 2002 Obesity in South Africa: The South African demographic and health survey. Obesity Research, 10, 1038-1048.

Puhl RM, \& Brownell KD 2001 Bias, discrimination, and, obesity. Obesity Research, 9, 788-805.

Rosmond R 2004 Aetiology of obesity: A striving after wind? Obesity Reviews, 5, 177-181.

Satta A 2000. Exercise training in asthma. Journal of Sports Medicine and Physical Fitness, 40, 277-283.

Snyder, EE, Walts B, Perusse L, Chagnon YC, Weisnagel SJ, Rankinen T \& Bouchard C 2004 The human obesity gene map: The 2003 update. Obesity Research, 12(3), 369-439.

Swift SA, Glazebrook C, \& Macdonald I 2006 A valid scale to measure knowledge about obesity-related health risks. International Journal of Obesity, 30, 661-668.
Swift JA, Sheard C, \& Rutherford M 2007 Trainee health care professionals' knowledge of the health risks associated with obesity. Journal of Human Nutrition \& Dietitian, 20, 599-604.

Thomas S, Hyde J, \& Komesaroff P 2007 Cheapening the struggle: Obese people's attitudes towards 'the biggest loser'. Obesity Management, 210-214.

U S Department of health and Human Services 2001. The surgeon-general's call to action to prevent and decrease overweight and obesity. Washington, DC: United States Government Printing Office.

World Health Organisation. 2006 Global strategy on diet, physical activity and health: Obesity and overweight. World Health Organisation. Retrieved from http://www.who.int/dietphysicalactivity/publications/facts/obesity/en/2006 